\title{
Grain Refinement by Vibration
}

\section{by J. L. Walker, General Electric Co.}

The use of vibration as a tool to control structures produced during solidification of ingots and castings has been the subject of considerable discussion recently. Although it does not appear that vibration can solve all of the grain size and shape problems encountered in ingot and foundry practice, it does provide the metallurgist with an additional means of grain size control. It has been reported by many investigators that vibration of the proper intensity transmitted either to the mold or introduced directly in the melt will produce grain refinement in a variety of alloys. Although the technique has been known and used for some time, there is still considerable diversity of opinion regarding the specific mechanism or mechanisms by which the introduction of sonic energy into a melt can increase the number of grains that form during solidification.

Some of the earlier workers tended to use the ultrasonic frequencies to produce structural changes in metals undergoing solidification. However, the work of Hinchliff and Jones ${ }^{1}$ indicated that effective grain refinement could be produced in a hightemperature alloy using subsonic frequencies. They also reported that increasing frequency rather than increasing amplitude had the most potent effect on grain size. Richards and Rostoker ${ }^{2}$ showed in an investigation of the effect of vibration on the solidification of an aluminum alloy that a plot of grain size vs displacement yields a frequency independent correlation. These somewhat conflicting results on different alloy systems amplify the need for a more fundamental understanding of the mechanism by which vibration effects solidification.

One of the earlier mechanisms proposed assumed that cavitation occurring in a melt under the influence of intense ultrasonic irradiation shattered dendrites producing showers of fresh nucleii for the formation of new crystals ${ }^{8}$. Direct observation of the crystallization of $\mathrm{NH}_{4} \mathrm{Cl}$ has indicated that the fragmentation hypothesis is unable to account for the grain refinement observed during vibration at sonic frequencies".

A more generally accepted mechanism by which vibration can pro- duce grain refinement has been described by Richards and Rostoker ${ }^{2}$. In this description it is hypothesized that vibration directly increases the nucleation rate. This increase in rate occurs presumably by the effect of pressure fluctuations on the size of the critical nucleus from which crystals grow. This mechanism has been discussed in some detail by Freedman and Wallace ${ }^{5}$ who point out that since the solid is usually more dense than the liquid, the LeChatelier principle would predict that the pressure wave of vibration would tend to promote solid state; i.e., increase the effective rate of nucleation. The magnitude of the pressure required to promote homogeneous nucleation at a sensible rate can be calculated by using the Clausius equation. It is assumed in the calculation that the effect of pressure is equivalent to that of lowering the temperature by an amount required to produce the supercooling at which nucleation will occur. One important conclusion drawn as a result of this kind of calculation is that either nucleation is heterogeneous or the pressure fluctuations produced by vibration are very large in magnitude; i.e., tens of thousands of atmospheres. Since pressures of this magnitude are not generated directly by the kinds of equipment used in vibration experiments, it follows that either vibration causes catastrophic cavitation which gives rise to very high pressures or nucleation occurs on catalysts accidentally included in the melt.

In general the grain size of a casting is determined by the thermal gradients during solidification; by the amount of alloying constituents available to promote constitutional super-cooling; and by the number, kind and distribution of potential nucleation catalysts available to promote nucleation. Any general hypothesis that rationalizes the effect of vibration on cast structure must consider its effect on all of these variables. For example, it might be expected that intense vibration will have a significant effect on the superheat and temperature gradients in the melt during solidification. Lane, Cunningham and Tiller ${ }^{\circ}$ have discussed the effect of ultrasonic radiation on solute distribution, temperature distribution, and nucleation frequency. They pointed out that any change in solute distribution could arise only through convective mixing of the liquid adjacent to the interface. Since convective mixing tends to reduce the magnitude of the constitutional supercooling the effect of vibration on constitutional supercooling is to reduce the probability of equiaxed grain formation. In considering the effect of ultrasonic radiation on the thermal gradient at a freezing interface, the authors pointed out that it is possible that the heat generated as a result of attenuation at the interface may be of sufficient magnitude to inhibit freezing while the superheat flows out through the interface. The thermal conditions in the casting during solidification would then be similar to those in castings poured with very little superheat, a condition that generally promotes grain refinement. The amount of acoustic energy required to produce the growth inhibiting effect is shown to be considerably higher than that required to produce grain refinement. Since it is not necessary to propose an effect of vibration on either solute or temperature gradients to explain their observations, Lane, Cunningham and Tiller concluded that the important effect of vibration involves nucleation.

A quantitative explanation of the precise mechanism by which vibration of a melt induces nucleation will depend upon the results of theoretical and experimental work now underway. This work is directed toward determining "the effect of pressure and turbulence on nucleation kinetics.

\section{References}

IS. Hinchliff and J. Jones: The Application of Subsonic Vibrations During Solidification of Castings with Particular Reference to a Material for Gas Turbine Blades, Crown Max, College Report no. 89, The College of Aeronautics, Cranfield Fing. 1955. 2 R. S. Richards and W. Rostoker: The Influence of Vibration on the Solidification of an Aluminum Alloy, Trans. ASM, vol. 48 1956, p. 884 .

3 A. E. Crawford: Ultrasonic Engineering, Butterworth Scientific Publications, London, 1955 , p. 233.

4 I. I. Novikov, G. A. Korol'kov, and V. S. Zolotorevskii: Izvest. VUZ-Chern. Metallurgiya, no. 5, May 1960, pp. 130-134.

5 A. Freedman and J.' Wallace: Vibrating Strength into Metals, Modern Castings, April 1957, p. 64.

${ }^{B} \mathrm{D}$. Lane, J. Cunningham, and $\mathbf{W}$. $A$ Tiller: The Application of Ultrasonic Energy to Ingot Solidification, AIME Trans., vol. 218 , no. 6, Dec. 1960 , p. 985. 